\title{
3 Research Square \\ FpgMBV1 Alleviates The Pathogenicity of Fusarium Pseudograminearum to Wheat Plants: A Megabirnavirus Case Study
}

\section{Yuan Xie}

Henan Agricultural University

\section{Zhifang Wang}

Henan Agricultural University

Ke Li

Henan Agricultural University

Dongwei Liu

Henan Agricultural University

Yifan Jia

Henan Agricultural University

Fei Gao

Henan Agricultural University

Junli Dai

Henan Agricultural University

Chao Zhang

Henan Agricultural University

Lin Zhou

Henan Agricultural University

Shengli Ding

Henan Agricultural University

Songbai Zhang

Yangtze University

Xiaoting Zhang ( $\nabla$ zhangxiaoting921@163.com )

Henan Agricultural University

Honglian Li

Henan Agricultural University

\section{Research}

Keywords: megabirnavirus, Fusarium pseudograminearum, Fusarium pseudograminearum megabirnavirus 1, hypovirulence, wheat crown rot disease 
Posted Date: December 9th, 2020

DOI: https://doi.org/10.21203/rs.3.rs-122141/v1

License: (c) (i) This work is licensed under a Creative Commons Attribution 4.0 International License. Read Full License 


\section{Abstract}

Background: Fusarium pseudograminearum is the predomenant causal agent of devastating crown rot diseases in cereal crops around the world. Mycoviruses have attracted increasing attention as potential biological control agents on plant diseases. The unique mycoviruse isolated from F. pseudograminearum is Fusarium pseudograminearum megabirnavirus 1 (FpgMBV1), which is a new member of the family Megabirnaviridae. To determine the hypovirulence effects of FpgMBV1 on F. pseudograminearum to wheat plants is critical for the potential application of FpgMBV1 in the control of cereal crown rot disease.

Methods: Hyphal tip cultures were conducted to obtain a FpgMBV1-free strain, named as FC136-2A-V . A hyg gene was transformed into a highly virulent virus-negative stain WZ-8A of F. pseudograminearum to

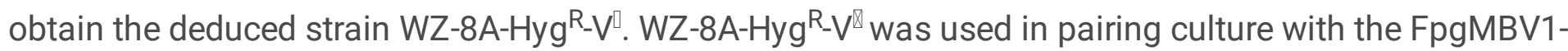
carrying F. pseudograminearum strain FC136-2A to obtain a FpgMBV1-positive strain WZ-8A-Hyg ${ }^{R}-V^{+}$. Then the two pairs of strains, FC136-2A-V $V^{\square} / F C 136-2 A$ and WZ-8A-Hyg ${ }^{R}-V^{\square} / W Z-8 A-H y g^{R}-V^{+}$, were used to determine the potential effect on F. pseudograminearum by the infection of FpgMBV1 through tests on the growth, sensitivity to stress and cellophane penetrating ability in vitro and the pathogenicity to wheat plants.

Results: FpgMBV1 could be cured by hyphal tip culture and horizontally transferred between $F$. pseudograminearum strains through pairing culture. Reduction of FpgMBV1-carrying strains on hyphal growth were found under the treatment of $0.5 \%$ SDS. No differences in the growth rates of tested strains in the treatments with $1 \mathrm{M} \mathrm{NaCl}, 1 \mathrm{M} \mathrm{KCl}$, or $15 \mathrm{mM} \mathrm{H}_{2} \mathrm{O}_{2}$. Comparing to virus-negative strains, the In vitro cellophane penetrating ability was lost in FpgMBV1-carrying strains. The disease index of wheat plants inoculated with FC136-2A-V $\mathrm{V}^{-}$was significantly higher than that inoculated with FC136-2A, while the pathogenicity of WZ-8A-Hyg ${ }^{R}-V^{+}$reduced significantly comparing to that of WZ-8A-Hyg ${ }^{R}-V^{\square}$.

Conclusions: FpgMBV1 is the causal agent of the low pathogenicity to wheat plants of its original host $F$. pseudograminearum strain FC136-2A. And FpgMBV1 could be horizontally transferred to another $F$. pseudograminearum strain and reduce its pathogenicity to wheat plants.

\section{Background}

Fusarium pseudograminearum, a soil-borne ascomycetous fungus, is the causal agent of wheat Fusarium crown rot (FCR). FCR is one of the most devastating fungal diseases in wheat crops in many countries or regions around the world. The typical disease symptoms on wheat were characterized by seedling blight, brown discoloration of the stem-bases with occasional pink hyphal gathered under the leaf sheaths, and whiteheads at maturity [1-3]. Besides the serious yield losses of about $60-85 \%$ annually in average in winter wheat [4-6], FCR disease may lead to wheat grain quality reduction due to the accumulation of mycotoxin trichothecenes nivalenol (NIV) and deoxynivalenol (DON) [7]. 
A series of viruses, referred to as mycoviruses, have been identified from major groups of fungi [8]. Some mycoviruses lead to the reduction of virulence of the plant-pathogenic fungi. Some of them have been successfully developed as biocontrol agent of plant disease. For instance, Cryphonectria parasitica hypovirus 1 (CHV1) has been used to control chestnut blight caused by Cryphonectria parasitica [9, 10]. In Fusarium species, more than 30 mycoviruses have been identified and fully sequenced [9-14]. 4 mycoviruses were associated with hypovirulence of the host Fusarium, and notably, 3 viruses were from F. graminearum [15-17]. Fusarium oxysporum f. sp. dianthi mycovirus 1 (FpgMBV1) can horizontally transfer from one compatible isolate to another, and vertically transfer to offspring accompanied by sporogenesis [11]. The FodV1-infected strain F. oxysporum f. sp. dianthi showed decreased colonizing efficiency when compared to the virus-negative strain [18]. Fusarium graminearum virus 1 (FgV1) serves as a hypovirulence-associated virus in DK21 strain, while FgV1-ch, another mycovirues shared $95.91 \%$ identity in genome sequence with FgV1-DK21, had mild or no visible effects on host virulence and colonial morphology [14]. Comparative transcriptome and proteome analysis had provided many insights into understanding the interaction between virus and its host as well as the underlying mechanism of the mycovirus-mediated hypovirulence in fungi $[19,20]$. Host factors like a hexagonal peroxisome gene FgHex1, a halotolerance gene FgHal2 and a transcription factor geneFgSWI6 (FGSG-04220) are found to be involved in the virus accumulation, host responses or phenotypic alterations in the interaction between FgV1-DK21 and F. graminearum [21, 22]. Vr1, a putative mRNA-binding protein encoding gene, is responsible for the symptom induction during FgV-ch9 infection [23]. The RNA interference (RNAi)associated defense responses of fungi to mycovirus, were differently activated by FgV1, FgV12 and FgV13 [24]. The transcription level of DICER-2 and AGO-1 genes of F. graminearum (FgDICER-2 and FgAGO-1) were suppressed in response to FgV1 infection [24]. This suppression was caused by the FgV1 pORF2, which binds to the upstream region of these two genes in vitro [25]. Many studies have been done on the hypovirulence of Chrysoviridae (FgV-ch9) and Fusariviridae (FgV1-DK21, FgV1-ch) in F. graminearum. However, little was known about how megabirnavirius FpgMBV1 regulates $F$. pseudograminearum pathogenicity.

To test whether the megabirnavirus FpgMBV1 plays a role in the hypovirulence of FC136-2A and regulation of the in vitro growth of FC136-2A, a hyphal tip culture and paired culture method was applied to cure and transmit FpgMBV1 from the F. pseudograminearum isolate FC136-2A. The pathogenicity of FpgMBV1-free and FpgMBV1-containing strains were tested by pathogenicity measurement on wheat. This is the first report on the hypovirulence of mycoviruses on F. pseudograminearum. The results extend our knowledge on mycoviruses, especially megabirnavirus and their potent application on the biological control of plant diseases.

\section{Methods}

Detection of mycoviruses by reverse transcriptional PCR. To confirm that the FpgMBV1 has been purged from the FC136-2A isolate and successfully transformed into the WZ-8A-Hyg ${ }^{\mathrm{R}}-\mathrm{V}^{+}, \mathrm{RT}-\mathrm{PCR}$ was performed with FpgMBV1-P3-F: 5'-CATGCCATGGATGTCAAGGC-3' and FpgMBV1-P3-R: 5'- 
CGGAATTCCTATTGCATTGCTATC-3'. The RT reaction solution was prepared as follows: $6 \mu$ of dsRNA, $5 \mu$ of $5 \times$ RT buffer, $2 \mathrm{mM}$ of dNTP (Takara, Japan) and $5 \mathrm{U}$ of Reverse Transcriptase M-MLV (RNase $\mathrm{H}^{-}$) (Takara, Japan). The reaction procedure was shown below: $25^{\circ} \mathrm{C} 5 \mathrm{~min}, 42^{\circ} \mathrm{C} 60 \mathrm{~min}$ and $75^{\circ} \mathrm{C} 5 \mathrm{~min}$. Next, $20 \mu$ PCR reaction solution was prepared as follows: $9.5 \mu$ of $2 \times$ Taq PCR StarMix with Loading Dye (GenStar, Biotechnology, Inc., China), $0.05 \mu \mathrm{M}$ forward primer, $0.05 \mu \mathrm{M}$ reverse, $2 \mu \mathrm{l}$ of cDNA and $7.5 \mu$ double distilled water $\left(\mathrm{ddH}_{2} \mathrm{O}\right)$. The PCR reaction solution was operated at $95^{\circ} \mathrm{C}$ for $5 \mathrm{~min}$, followed by 35 cycles of $95^{\circ} \mathrm{C}$ for $30 \mathrm{~s}, 58^{\circ} \mathrm{C}$ for $30 \mathrm{~s}$ and $72^{\circ} \mathrm{C}$ for 1 min and then a final extension at $72{ }^{\circ} \mathrm{C}$ for 10 min. Finally, PCR products stained with StarStain Red Nucleic Acid Dye (GenStar, Biotechnology, Inc., China) were analyzed in a $1 \%$ agarose gel by electrophoresis. The amplified fragments were photographed using an agarose gel imaging system (Bio-Rad Laboratories, Inc., America).

In vitro growth of the fungi in PDA medium. An in vitro culture experiment of the fungal colony was performed to analyze the developmental phenotypes of both isolates, including the original strain FC136$2 \mathrm{~A}$ carrying FpgMBV1 and virus-negative strain FC136-2A- $\mathrm{V}^{-}$. First, stock culture of both strains was reactivated on a $15 \mathrm{ml}$ PDA Petri plates in a biochemical incubator at $25^{\circ} \mathrm{C}$ for five days. A $5 \mathrm{~mm}$ diameter mycelia disk from each reactivated culture was sown in the center of $15 \mathrm{ml}$ PDA Petri plates and incubated again in a biochemical incubator at $25^{\circ} \mathrm{C}$. The growth rate on each Petri plate (largest and smallest colony diameter) was measured each day until one strain completely covered the dish. Five biological replicates were performed in the experiment and each biological replicate was repeated three times. The analysis of variance (ANOVA) with the application of a split-plot design and Duncan's assumption of homoscedasticity $(p<0.05)$ was used to analyze the data in the software Statistical Product and Service Solutions (SPSS; Version 20.0).

Preparation of the recipient F. pseudograminearum strains with FpgMBV1. The recipient $F$. pseudograminearum strain $\mathrm{WZ}-8 \mathrm{~A}-\mathrm{Hyg}^{\mathrm{R}}-\mathrm{V}^{-}$was a transformant strain expressing a hygromycin resistant $(H p h)$ gene and a green fluorescent protein (GFP) gene through agrobacterium-mediated gene transformation. Briefly, the fresh single colonies of A. tumefaciens containing PCAM-GFP-hyg plasmid were picked and put in $10 \mathrm{ml} \mathrm{LB}$ liquid medium (kanamycin $50 \mathrm{mg} \cdot \mathrm{L}^{-1}$ ) to be shake cultured overnight at $28{ }^{\circ} \mathrm{C}$ until the $\mathrm{OD}_{600}$ of the bacterial liquid reached at 0.6-0.8. Then, $400 \mu \mathrm{l}$ of the cultured liquid was transferred into $10 \mathrm{ml}$ induction medium (IM) and shaken at $28{ }^{\circ} \mathrm{C}$ at $220 \mathrm{rpm}$ for about $6 \mathrm{~h}$ until the $\mathrm{OD}_{600}$ of the bacterial liquid reached at 0.2 . The colony plugs of the wild-type $F$. pseudograminearum strain WZ-8A were put in carboxymethylcellulose sodium fluid medium (CMC) under the conditions of shake-flask culture at $25^{\circ} \mathrm{C}$ at $150 \mathrm{rpm}$ for $4-5$ days. The cultured suspension was filtered with doublelayer membrane and centrifuged at $3500 \mathrm{rpm}$ at $4{ }^{\circ} \mathrm{C}$ for $6 \mathrm{~min}$. The precipitates containing the conidia of WZ-8A were washed with sterile water and stored at $4{ }^{\circ} \mathrm{C}$. The prepared conidial suspension of WZ-8A was then mixed with the cultured agrobacterium liquid until the final concentration of the conidial suspension was around $10^{6}$ cells per milliliter. $200 \mu$ of mixed liquid was spread on co-culture medium (CO-IM) covered with nitrocellulose membrane and cultured at $25^{\circ} \mathrm{C}$ for 2 days. Then the nitrocellulose membrane was inverted onto the selective medium containing hygromycin $\left(50 \mathrm{mg} \cdot \mathrm{L}^{-1}\right)$ and 
cephalosporin ( $400 \mathrm{mg} \cdot \mathrm{L}^{-1}$ ) and cultured in $25^{\circ} \mathrm{Cfor} 4$ days. Then the nitrocellulose membrane was removed, and the single colonies were prepared for future study.

Horizontal transmission of hypovirulence. To determine whether the hypovirulence trait of $F$. pseudograminearum FC136-2A was associated with FpgMBV1, and FpgMBV1 can transmit through hyphal anastomosis, pairing culture experiments were conducted. The hypovirulence strain FC136-2A containing FpgMBV1 virus-negative stain WZ-8A-Hyg ${ }^{R}$-V'were used as the donor strain and recipient strain, respectively. Mycelial plugs of the donor and recipient combinations were individually cultured $1 \mathrm{~cm}$ apart on a PDA dish at $25^{\circ} \mathrm{C}$ until the mycelium from both isolates came in contact. Mycelial agar plugs were taken from the edge of the recipients' colony and transferred to PDA plates with hygromycin $\left(75 \mathrm{mg} \cdot \mathrm{L}^{-1}\right)$. The grown colonies were subjected to single conidia selection in water-agar (WA) plates to obtain recipient derivative isolates.

Detection of the sensitivity to environmental stress in vitro. The F. pseudograminearum strains were inoculated with mycelial blocks using a perforator $\left(0.5 \mathrm{~cm}\right.$ in diameter) in PDA medium at $25^{\circ} \mathrm{C}$ in the dark for 4 days by individually adding $1 \mathrm{M} \mathrm{NaCl}, 1 \mathrm{M} \mathrm{KCl}, 0.5 \%$ SDS or $15 \mathrm{mM} \mathrm{H}_{2} \mathrm{O}_{2}$, and incubated at $25^{\circ} \mathrm{C}$ in the dark for 4 days. The colonial morphology of these strains was record and the growth rate was measured with the crossover method.

In vitro cellophane penetrating experiment. One layer of cellophane was put on each PDA medium. Then after growing 2 days in the medium at $25^{\circ} \mathrm{C}$ in the dark, the hyphal were moved away with the cellophane. The leftover PDA plates were incubated for another 2 days at $25^{\circ} \mathrm{C}$ in the dark and took picture.

Pathogenicity test of F. pseudograminearum strains on wheat plants. The susceptible wheat cultivar Aikang 58 was used in this experiment and grown in a greenhouse with $25^{\circ} \mathrm{C}$ and a humidity of $60 \%$. The inoculation of FC136-2A, FC136-2A-V $\mathrm{V}^{-}$, WZ-8A-Hyg ${ }^{\mathrm{R}}-\mathrm{V}^{-}, \mathrm{WZ}-8 \mathrm{~A}-\mathrm{Hyg} \mathrm{g}^{\mathrm{R}}-\mathrm{V}^{+}$was individually performed as described previously [26]. The non-inoculated plants were used as empty controls. All the isolates were grown on PDA to prepare the mycelia plugs for inoculum. Then the mycelial plugs were used to inoculate into the autoclaved millet seeds in triangular flasks at $25^{\circ} \mathrm{C}$ for $4-6$ days until the seeds were extensively colonized. Ten surface-disinfected seeds were sown in potsfilled with sterilized soil mixed with $1 \mathrm{~g}$ colonized millet. Three independent experiments were done with four plastic pots of plants for each fungal isolate. The disease severity index (DSI) values were calculated 15 days after inoculation using a 0 to 7 rating scale $[26,27]$. Data were analyzed with the analysis of variance (ANOVA) using a split-plot design and Duncan's assumption of homoscedasticity $(p<0.05)$ in the software Statistical Product and Service Solutions (SPSS; Version 20.0).

\section{Results}

FpgMBV1 curing through hyphal tip culture. Through hyphal tip culture and dsRNA detection, we obtained a strain without existence of FpgMBV1, designated as FC136-2A-V ${ }^{-}$(Fig. 1). As shown in Fig. 2A, no 
visible developmental abnormalities were found in $\mathrm{FC} 136-2 \mathrm{~A}-\mathrm{V}^{-}$strain compared to the virus-containing FC136-2A strain. Virus detection was marked by the dsRNA electrophoretic analysis. Results showed that FpgMBV1 is absent in FC136-2A- $\mathrm{V}^{-}$, while strong signals of the dsRNA genome of FpgMBV1 was found in FC136-2A (Fig. 2B).

The presence of FpgMBV1 in FC136-2A was further confirmed by reverse transcription-PCR. As expected, the 2700 bp DNA fragment of FpgMBV1 is undetectable from FC136-2A- $\mathrm{V}^{-}$strain, but it is detected from FC136-2A (Fig. 2C).

FpgMBV1 can horizontally transfer to virus-negative strains through hyphal fusion. To indicate the presence of viral dsRNA in the recipient isolate WZ-8A-Hyg ${ }^{R}-V^{-}$, the available monoconidial offspring cultures were collected to conduct dsRNA purification analyses by cellulose column chromatography as described previously [17]. We found that some of the WZ-8A-Hyg ${ }^{R}-V^{\natural}$ monoconidial offspring cultures analyzed possessed dsRNA segments, which is in line with those extracted from the donor isolate FC1362A (Fig. 3B). To prove that these dsRNA segments is a part of the genome of FpgMBV1, total RNA extracts were obtained using TRIzol ${ }^{\circledR}$ Reagent (Ambion ${ }^{\circledR}$, Thermo Fisher Scientific, Waltham, MA USA) and then used to perform a reverse transcription-PCR using specific primers for the ORF3 sequence of FpgMBV1. DNA extracts were obtained using CTAB method and then used to perform a PCR using specific primers for Hyg and GFP genes in the donor strains (Fig. 3C). These FpgMBV1 positive monoconidial cultures were designated as WZ-8A-Hyg ${ }^{\mathrm{R}}-\mathrm{V}^{+}$. No alteration in the colony morphology and growth rates were found for WZ-8A-Hyg ${ }^{R}-V^{+}$(Fig. 3A)

Sensitivity to environmental stress in vitro. Under normal conditions, all the four strains, including FC1362A, FC136-2A-V $\mathrm{V}^{-}, \mathrm{WZ}-8 \mathrm{~A}-\mathrm{Hyg}^{\mathrm{R}}-\mathrm{V}^{-}$and $\mathrm{WZ}-8 \mathrm{~A}-\mathrm{Hyg}^{\mathrm{R}}-\mathrm{V}^{+}$, exhibited no difference in growth rate as well as the colonial morphology. Also, individually treatment of all the four strains with $1 \mathrm{M} \mathrm{NaCl}, 1 \mathrm{M} \mathrm{KCl}, 0.5 \% \mathrm{SDS}$ or $15 \mathrm{mM} \mathrm{H}_{2} \mathrm{O}_{2}$ were performed. Reduction of FpgMBV1-carrying strains, FC136-2A and WZ-8A-Hyg ${ }^{\mathrm{R}}-\mathrm{V}^{+}$, on hyphal growth were found under the treatment of $0.5 \%$ SDS, but no significant differences between that of virus-positive strains and virus-negative strains (Fig. 4). No alterations for other treatments. These findings suggested that cell wall sensitivity to environmental stress factors of $F$. pseudograminearum were disturbed by FpgMBV1.

In vitro cellophane penetrating experiments. Results showed that no penetrated colony was found for WZ-

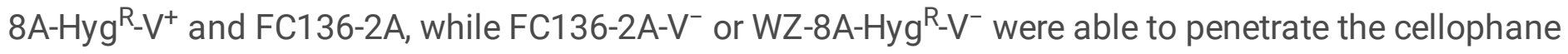
and form obvious new colony 2 days after pealing of the cellophane (Fig. 5A). That means FpgMBV1 may destroyed the infection structures of its host fungi.

FpgMBV1 reduces fungi virulence in wheat plants. As shown in Fig. 5B, severe disease symptom like necrosis on the stem base of wheat seedlings was found in plants inoculated with $\mathrm{FC} 136-2 \mathrm{~A}-\mathrm{V}^{-}$strain, compared to the plants inoculated with virus-containing FC136-2A strain. The lesion length on wheat seedlings inoculated with either $\mathrm{FC} 136-2 \mathrm{~A}-\mathrm{V}^{-}$or $\mathrm{WZ}-8 \mathrm{~A}-\mathrm{Hyg}^{\mathrm{R}}-\mathrm{V}^{-}$is much shorter than that inoculated with 
WZ-8A-Hyg ${ }^{\mathrm{R}}-\mathrm{V}^{+}$and FC136-2A. In addition, the disease index showed significantly higher values in plants inoculated with FC136-2A- $\mathrm{V}^{-}$than $\mathrm{FC136-2A}$ (Fig. 5C). Similar results were found in plants inoculated with WZ-8A-Hyg ${ }^{\mathrm{R}}-\mathrm{V}^{-}$, compared to that inoculated with WZ-8A-Hyg ${ }^{\mathrm{R}}-\mathrm{V}^{+}$. These findings indicated that FpgMBV1 can markedly reduce the pathogenicity of $F$. pseudograminearum on wheat seedlings.

\section{Discussion}

Hyphal tip cultures have been proved applicable for different virus/host systems [28]. In our study, hyphal tip cultures were successfully used to cure F. pseudograminearum isolate FC136-2A of a megabirnavirus, FpgMBV1. The elimination of FpgMBV1 from FC136-2A allowed us to test biological effects of FpgMBV1 on the fungal host. Results showed that FpgMBV1 is associated with hypovirulence and there is no reduction in in vitro growth of the F. pseudograminearum strains (Figs. 2 and 5).

Hypovirulence effects were not always along with the changes on biological properties of the host fungi strains. Some megabirnaviruses confer hypovirulence to their hosts as well as the perturbation of growth. For instance, Rosellinia necatrix megabirnavirus 1 (RnMBV1) was responsible for the reduction of both virulence and mycelial growth in several host strains of Rosellinia necatrix [29]. Some megabirnaviruses have slight or no impact on both the biological properties and virulence of its hosts, such as Rosellinia necatrix megabirnavirus 2 (RnMBV2) to $R$. necatrix and Sclerotinia sclerotiorum megabirnavirus 1 (SsMBV1) to Sclerotinia sclerotiorum isolate SX466 [30]. Besides, the hypovirulence effects on host fungi of megabirnaviruses may change. For example, RnMBV1 showed significant attenuation on the virulence of $C$. parasitica EP155, with no reduction on the colony growth rates [31]. RnMBV2 exhibited hypovirulence effects on $R$. necatrix when co-infecting with Rosellinia necatrix partitivirus 1 (RnPV1) [32]. To verify the linkage between the hypovirulence effects of FpgMBV1 and the biological changes of its host fungi strains, FpgMBV1 was horizontally transferred to a virus-negative F. pseudograminearum strain WZ-8A-Hyg ${ }^{R}-V^{-}$in this study. The hypovirulence effects were transferred simultaneously to the new obtained strains. Both the original and the new obtained strains infected by FpgMBV1 showed no significant changes on biological traits including colonial morphology, hyphal growth in vitro and osmotic and oxidative stress tolerance. Further studies would be done to test the effects of FpgMBV1 on other potential host fungi.

During the horizontal transfer of FpgMBV1, no observation of the lost or rearrangement of FpgMBV1 genome dsRNAs appeared. But for other megabirnaviruses like RnMBV1, SsMBV1 and RnMBV2, the second dsRNA segment could be lost when the viruses were horizontally transferred from one strain to another [30-32]. For SsMBV1, L2-dsRNA/SsMBV1 was lost in two-thirds of the derivatives based on both the horizontal transmission assay and virion transfection assays [30]. And L2-dsRNA/SsMBV1 was dispensable for SsMBV1 replication and packaging. For RnMBV1, dsRNA2/RnMBV1 was lost during transfections of $R$. necatrix with purified RnMBV1 virions and in 2 strains out of 25 strains of $C$. parasitica transfected. The mutant virus in $R$. necatrix, namely RnMBV1-RS1, contained dsRNA1 and a newly emerged dsRNAS1 segment, but lost dsRNA2. DsRNA2/RnMBV1 was shown to be dispensable for virus replication but necessary to hypovirulence of $R$. necatrix. Similar phenomena were found in RnMBV2. 
FpgMBV1 were transmitted from donor strain FC136-2A to recipient strain WZ-8A-Hyg ${ }^{\mathrm{R}}-\mathrm{V}^{-}$stably maintained with the constant two genome segments in the horizontal transmission assay in this study (Fig. 3). But it would be interesting to test whether L2-dsRNA /FpgMBV1 could be lost by vertical transmission and is dispensable for virus replication and the hypovirulence of F. pseudograminearum.

The hypovirulence phenomena of host fungi depends on the cooperation results of virus genes and its host partner. Commonly, there are two ORFs for megabirnavirus dsRNA2s (ORF3 and ORF4 in RnMBV1, RnMBV2 and FpgMBV1, ORF A and ORF B in SsMBV1). But only the ORF3-coded proteins, not the ORF4coded proteins of different megabirnaviruses, had sequence similarity. The ORF3-coded protein of RnMBV1 and SsMBV1 was suggested to be involved in efficient replication of viruses [30, 31]. Antibodies against full length putative ORF3 proteins detected only the $40 \mathrm{kDa}$ protein from $R$. necatrix strains infected with RnMBV1 [31]. A complex detection pattern with at least three protein bands corresponding to molecular masses of 150, 30 and $23 \mathrm{kDa}$ were observed specifically in RnMBV1-infected mutant strain $\Delta d c /$-2 of $C$. parasitica. As for FpgMBV1, antibodies against recombinant ORF3/FpgMBV1-encoded protein produced in Escherichia coli detected the $90 \mathrm{kDa}$ protein as the major protein in the hyphae of FpgMBV1 positive F. pseudograminearum strains [33]. How the ORF3-coded protein involved in the virus replication process and maybe the hypovirulence to its host fungi, will be studied further.

On the host fungi side, in vitro experiments showed that FpgMBV1 reduced the cellophane penetrating ability of host fungi strains (Fig. 5A). The underlying mechanisms on the formation of infection structures and gene expressions would be studied further. For other megabirnaviruses, RnMBV1 were proved to activate the expression of RNA silencing related genes [34, 35]. Besides, 1160 genes in $R$. necatrix were found to be differently expressed during infection of RnMBV1 [36]. The involvement of RNA silencing pathways and pathogenic related genes of $F$. pseudograminearum in the hypovirulence of FpgMBV1 remains unknown. Addressing these problems would probably advance the knowledge on the understanding and usage of mycoviruses conferring hypovirulence on important plant pathogenic fungi.

As we all know, many Fusarium species exist as complex in the soil. Megabirnavirus like RnMBV1 was successfully transferred to an artificial host $C$. parasitica. It would be interesting to test the horizontal transfection of FpgMBV1 from F. pseudograminearum into other fungi including other Fusarium species, especially some pathogenic species like F. graminearum, F. verticillioides, F. oxysporium etc. The ecological environments in the soil play a decisive role in plant disease control. To clarify the host range of mycoviruses would lay solid foundations to the mycoviruses application.

\section{Conclusions}

FpgMBV1 could be cured by hyphal tip culture and horizontally transferred between $F$. pseudograminearum strains through pairing culture. FpgMBV1 is the causal agent of the low pathogenicity to wheat plants of its original host F. pseudograminearum strain FC136-2A. And FpgMBV1 could be horizontally transferred to another $F$. pseudograminearum strain and reduce its pathogenicity to wheat plants. 


\section{Abbreviations}

bp: Base pair

CHV1: Cryphonectria parasitica hypovirus 1

cm: Centimeter

CMC: Carboxymethylcellulose sodium fluid medium

CO-IM: Co-culture induction medium

CTAB: Hexadecyl trimethyl ammonium Bromide

$\mathrm{ddH}_{2} \mathrm{O}$ : Double distilled water

DNA: Deoxyribonucleic acid

DON: Deoxynivalenol

DSI: Disease severity index

dsRNA: Double-stranded RNA

FCRQFusarium crown rot

FgHal2: Fusarium graminearum halotolerance 1 gene

FgHex1: Fusarium graminearum hexagonal peroxisome 1 gene

FgV1: Fusarium graminearum virus 1

FgV12: Fusarium graminearum virus 12

FgV13: Fusarium graminearum virus 13

FgV1-ch: Fusarium graminearum virus 1 Chinese isolate

FgV1-DK21: Fusarium graminearum virus 1 strain DK21

FgV-ch9: Fusarium graminearum virus China 9

FpgMBV1囚Fusarium pseudograminearum megabirnavirus 1

FpgMBV1-P3囚Fusarium pseudograminearum megabirnavirus 1 protein3

g: Gram 
GFP: Green fluorescent protein

IM: Induction medium

h: Hour

Hyg: Hygromycin resistance gene

kb: Kilobyte

M: Molar

mg: Milligram

min: Minute

ml: Millilitre

mM: Millimolar

NIV『Nivalenol

ORF: Open reading frame

PCR: Polymerase chain reaction

PDA: Potato-dextrose agar

pORF2: ORF2-encoded protein

RNA: Ribonucleic acid

RNAi: RNA interference

RnMBV1: Rosellinia necatrix megabirnavirus 1

RnMBV2: Rosellinia necatrix megabirnavirus 2

RnPV1: Rosellinia necatrix partitivirus 1

rpm: Rounds per minute

RT-PCR: Reverse transcription and a polymerase chain reaction

SPSS: Statistical Product and Service Solutions

SsMBV1: Sclerotinia sclerotiorum megabirnavirus 1 
ssRNA: Single stranded RNA

WA: Water agar

\section{Declarations}

\section{Ethics approval and consent to participate}

Not applicable.

\section{Consent for publication}

Not applicable.

\section{Availability of data and materials}

The datasets used and/or analysed during the current study are available from the corresponding author on reasonable request.

All data generated or analysed during this study are included in this published article.

\section{Competing interests}

The authors declare that they have no competing interests.

\section{Funding}

This work was financially supported by the Major International (Regional) Joint Research NSFC-CGIAR Project (31961143018), Henan Province Agricultural Science and Technology Research Projects (182102110004), Henan Province Young Core Teacher in University Support Project (2018GGJS032) and Henan Agricultural University Science and Technology Innovation Funding (KJCX2020A14).

\section{Authors' contributions}

YX performed the examination of the pathogenicity and the sensitivity of $F$. pseudograminearum to environmental stress in vitro and was a major contributor in drafting the manuscript.

ZFW prepared the recipient $F$. pseudograminearum strains with FpgMBV1.

$\mathrm{KL}$ performed the detection of mycoviruses by reverse transcriptional PCR.

DWL performed the horizontal transmission of hypovirulence.

YFJ performed the in vitro culture experiment of the fungi in PDA medium. 
FG statistically analyzed and interpreted the data of pathogenicity test of $F$. pseudograminearum strains on wheat plants and supported with the source of funding Henan Province Agricultural Science and Technology Research Projects (182102110004).

JLD interpreted the data regarding the detection of the sensitivity to environmental stress in vitro.

SBZ assisted with the preparation of the manuscript content.

CZ was a contributor in revising the manuscript.

LZ was a contributor in revising the manuscript.

SLD was a contributor in revising the manuscript.

XTZ designed the work and drafted the manuscript and supported with the source of funding Henan Province Young Core Teacher in University Support Project (2018GGJS032) and Henan Agricultural University Science and Technology Innovation Funding (KJCX2020A14).

HLL was a major contributor in revising the manuscript and supported with the source of funding Major International (Regional) Joint Research NSFC-CGIAR Project (31961143018).

All authors read and approved the final manuscript.

\section{Acknowledgements}

Not applicable.

\section{Footnotes}

Not applicable.

\section{References}

1. Wallwork H. Cereal Root and Crown Diseases. 2000;

2. Dyer AT, Johnston RH, Hogg AC, Johnston JA. Comparison of pathogenicity of the Fusarium crown rot (FCR) complex (F. culmorum, F. pseudograminearum and F. graminearum) on hard red spring and durum wheat. Eur J Plant Pathol. 2009;125:387-95.

3. Hollaway GJ, Exell GK. Survey of wheat crops for white heads caused by crown rot in Victoria, 19972009. Australas Plant Pathol. 2010;39:363-7.

4. Paulitz TC, Smiley RW, Cook RJ. Insights into the prevalence and management of soilborne cereal pathogens under direct seeding in the Pacific Northwest, U.S.A. Can J Plant Pathol. 2002;24:416-28.

5. Smiley RW, Whittaker RG, Gourlie JA, Easley SA, Ingham RE. Plant-parasitic nematodes associated with reduced wheat yield in Oregon: Heterodera avenae. J Nematol. 2005;37:297-307. 
6. Hollaway GJ, Evans ML, Wallwork H, Dyson CB, Mckay AC. Yield loss in cereals, caused by Fusarium culmorum and F. pseudograminearum, is related to fungal DNA in soil prior to planting, rainfall, and cereal type. Plant Dis. 2013;97:977-82.

7. Mudge AM, Dill-Macky R, Dong Y, Gardiner DM, White RG, Manners JM. A role for the mycotoxin deoxynivalenol in stem colonisation during crown rot disease of wheat caused by Fusarium graminearum and Fusarium pseudograminearum. Physiol Mol Plant Pathol. 2006;69:73-85.

8. Ghabrial SA, Castón JR, Jiang D, Nibert ML, Suzuki N. 50-Plus years of fungal viruses. Virology. 2015;479-480:356-68.

9. Nuss DL. Biological control of chestnut blight: An example of virus-mediated attenuation of fungal pathogenesis. Microbiol Rev. 1992;56:561-76.

10. Dawe AL, Nuss DL. Hypoviruses and chestnut blight: Exploiting viruses to understand and modulate fungal pathogenesis. Annu Rev Genet. 2001;35:1-29.

11. Lemus-Minor CG, Cañizares MC, García-Pedrajas MD, Pérez-Artés E. Horizontal and vertical transmission of the hypovirulence-associated mycovirus Fusarium oxysporum f. sp. dianthi virus 1. Eur J Plant Pathol. 2019;153:645-50.

12. Torres-Trenas A, Cañizares MC, García-Pedrajas MD, Pérez-Artés E. Molecular and Biological Characterization of the first hypovirus identified in Fusarium oxysporum. Front Microbiol. 2020;10.

13. Yao Z, Zou C, Peng N, Zhu Y, Bao Y, Zhou Q, et al. Virome Identification and Characterization of Fusarium sacchari and F. andiyazi: Causative agents of Pokkah boeng Disease in Sugarcane. Front Microbiol. 2020;11.

14. Zhang L, Chen X, Bhattacharjee P, Shi Y, Guo L, Wang S. Molecular characterization of a novel strain of Fusarium graminearum virus 1 infecting Fusarium graminearum. Viruses. 2020;12.

15. Lee KM, Yu J, Son M, Lee YW, Kim KH. Transmission of Fusarium boothii mycovirus via protoplast fusion causes hypovirulence in other phytopathogenic fungi. PLoS One. 2011;6.

16. Darissa O, Adam G, Schäfer W. A dsRNA mycovirus causes hypovirulence of Fusarium graminearum to wheat and maize. Eur J Plant Pathol. 2012;134:181-9.

17. Lemus-Minor CG, Canizares MC, Garciá-Pedrajas MD, Pérez-Artés E. Fusarium oxysporum f. sp. dianthi virus 1 accumulation is correlated with changes in virulence and other phenotypic traits of its fungal host. Phytopathology. 2018;108:957-63.

18. Torres-Trenas A, Prieto P, Cañizares MC, García-Pedrajas MD, Pérez-Artés E. Mycovirus Fusarium oxysporum f. sp. dianthi virus 1 decreases the colonizing efficiency of its fungal host. Front Cell Infect Microbiol. 2019;9.

19. Kwon SJ, Lim WS, Park SH, Park MR, Kim KH. Molecular characterization of a dsRNA mycovirus, Fusarium graminearum virus-DK21, which is phylogenetically related to hypoviruses but has a genome organization and gene expression strategy resembling those of plant potex-like viruses. Mol Cells. 2007;23:304-15.

20. Cho WK, Yu J, Lee KM, Son M, Min K, Lee YW, et al. Genome-wide expression profiling shows transcriptional reprogramming in Fusarium graminearum by Fusarium graminearum virus 1-DK21 
infection. BMC Genomics. 2012;13.

21. Yu J, Lee KM, Son M, Kim KH. Effects of the deletion and over-expression of Fusarium graminearum gene FgHal2 on host response to mycovirus Fusarium graminearum virus 1. Mol Plant Pathol. 2015;16:641-52.

22. Son M, Lee Y, Kim KH. The transcription cofactor swi6 of the Fusarium graminearum is involved in Fusarium graminearum virus 1 infection-induced phenotypic alterations. Plant Pathol J. 2016;32:281-9.

23. Bormann J, Heinze C, Blum C, Mentges M, Brockmann A, Alder A, et al. Expression of a structural protein of the mycovirus FgV-ch9 negatively affects the transcript level of a novel symptom alleviation factor and causes virus infection-like symptoms in Fusarium graminearum . J Virol. 2018;92.

24. Yu J, Lee K-M, Cho WK, Park JY, Kim K-H. Differential contribution of RNA interference components in response to distinct Fusarium graminearum virus infections. J Virol. 2018;92:e01756-17.

25. Yu J, Park JY, Heo Jl, Kim KH. The ORF2 protein of Fusarium graminearum virus 1 suppresses the transcription of FgDICER2 and FgAG01 to limit host antiviral defences. Mol Plant Pathol. 2020;21:230-43.

26. Zhou H, He X, Wang S, Ma Q, Sun B, Ding S, et al. Diversity of the Fusarium pathogens associated with crown rot in the Huanghuai wheat-growing region of China. Environ Microbiol. 2019;21:274054.

27. Smiley RW, Gourlie JA, Easley SA, Patterson LM. Pathogenicity of fungi associated with the wheat crown rot complex in Oregon and Washington. Plant Dis. 2005;89:949-57.

28. Tran TT, Li H, Nguyen DQ, Jones MGK, Wylie SJ. Co-infection with three mycoviruses stimulates growth of a monilinia fructicola isolate on nutrient medium, but does not induce hypervirulence in a natural host. Viruses. 2019;11.

29. Chiba S, Salaipeth L, Lin Y-H, Sasaki A, Kanematsu S, Suzuki N. A novel bipartite double-stranded RNA mycovirus from the white root rot fungus Rosellinia necatrix. Molecular and Biological Characterization, Taxonomic Considerations, and Potential for Biological Control. J Virol. 2009;83:12801-12.

30. Wang M, Wang Y, Sun X, Cheng J, Fu Y, Liu H, et al. Characterization of a novel megabirnavirus from Sclerotinia sclerotiorum reveals horizontal gene transfer from single-stranded RNA virus to doublestranded RNA virus. J Virol. 2015;89:8567-79.

31. Kanematsu S, Shimizu T, Salaipeth L, Yaegashi H, Sasaki A, Ito T, et al. Genome rearrangement of a mycovirus Rosellinia necatrix megabirnavirus 1 affecting its ability to attenuate virulence of the host fungus. Virology. 2014;450-451:308-15.

32. Sasaki A, Nakamura H, Suzuki N, Kanematsu S. Characterization of a new megabirnavirus that confers hypovirulence with the aid of a co-infecting partitivirus to the host fungus, Rosellinia necatrix. Virus Res. 2016;219:73-82. 
33. Li, K., Liu, D. W., Wang, Z. F., Xie, Y., Zhang, X. T. and Li HL. Prokaryotic expression of FpgMBV1-P3 and preparation of its polyclonal antibodies. Acta Phytopathol Sin. 2019;50:567-73.

34. Yaegashi H, Yoshikawa N, Ito T, Kanematsu S. A mycoreovirus suppresses RNA silencing in the white root rot fungus, Rosellinia necatrix. Virology. 2013;444:409-16.

35. Yaegashi H, Shimizu T, Ito T, Kanematsu S. Differential inductions of RNA silencing among encapsidated double-stranded RNA mycoviruses in the white root rot fungus Rosellinia necatrix. J Virol. 2016;90:5677-92.

36. Shimizu T, Kanematsu S, Yaegashi H. Draft genome sequence and transcriptional analysis of Rosellinia necatrix infected with a virulent mycovirus. Phytopathology. 2018;108:1206-11.

\section{Figures}

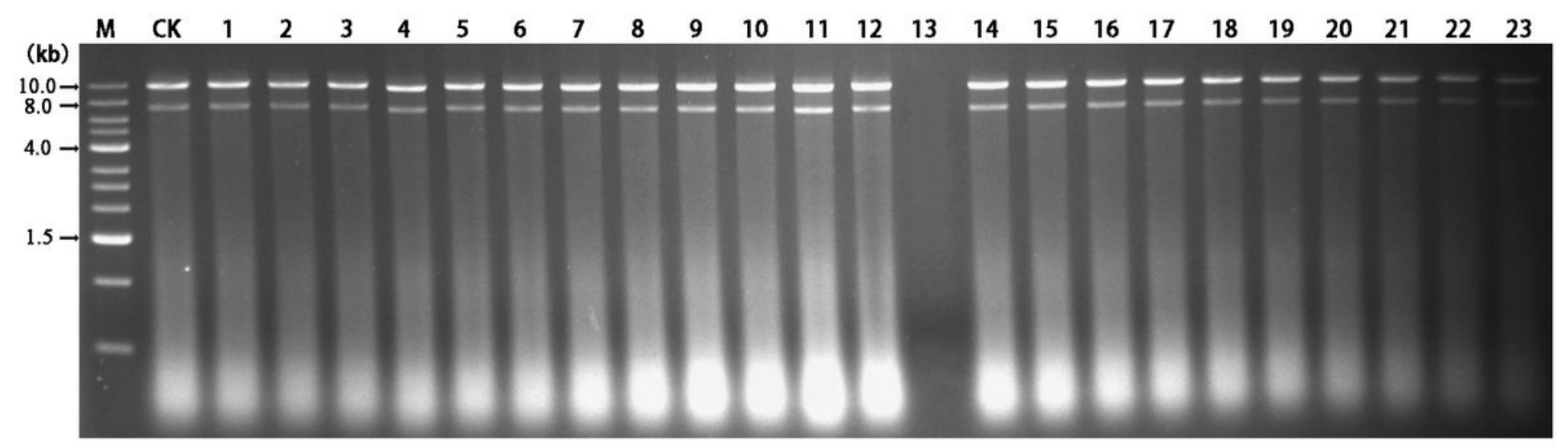

\section{Figure 1}

Detection of FpgMBV1 curing strains through hyphal tip culture by agarose gel electrophoresis of dsRNA fractions Lane M:1kb DNA Ladder Marker (TSINGKE); CK: FC136-2A; 1-23: Strains obtained by hyphal tip culture.

A

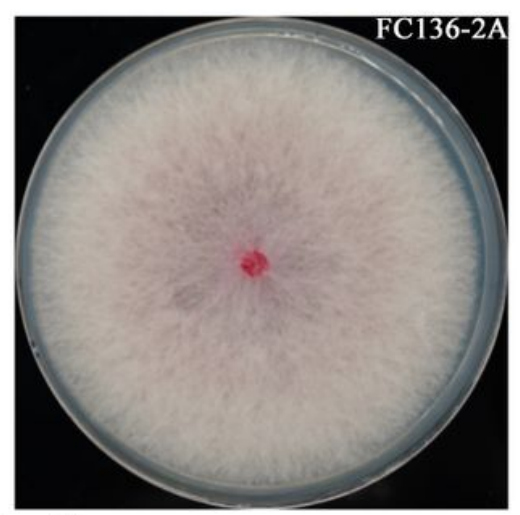

Growth $\operatorname{rate}(\mathrm{cm} / \mathrm{d})$

$2.04 \pm 0.04$

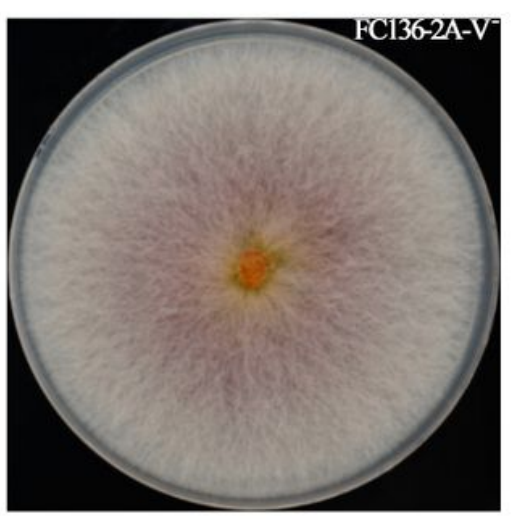

$1.96 \pm 0.03$
B

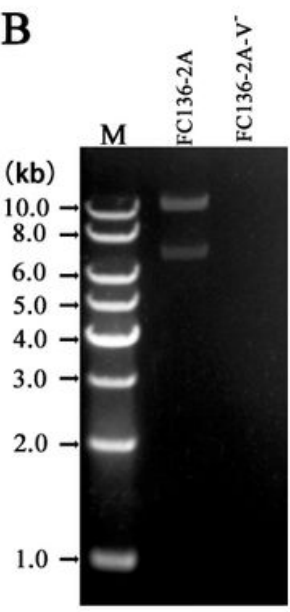

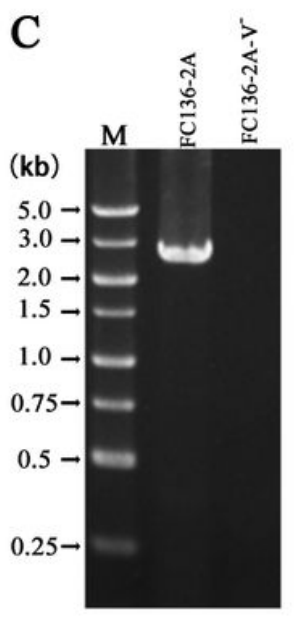

Figure 2 
Colonial morphology (A), agarose gel electrophoresis of dsRNA fractions (B) and RT-PCR amplification using FpgMBV1-P3 specific primers (C). Lane M: DNA Ladder (TRANSGEN); FC136-2A: FpgMBV1 positive F. pseudograminearum strain; FC136-2A-V-: FpgMBV1 cured strain.

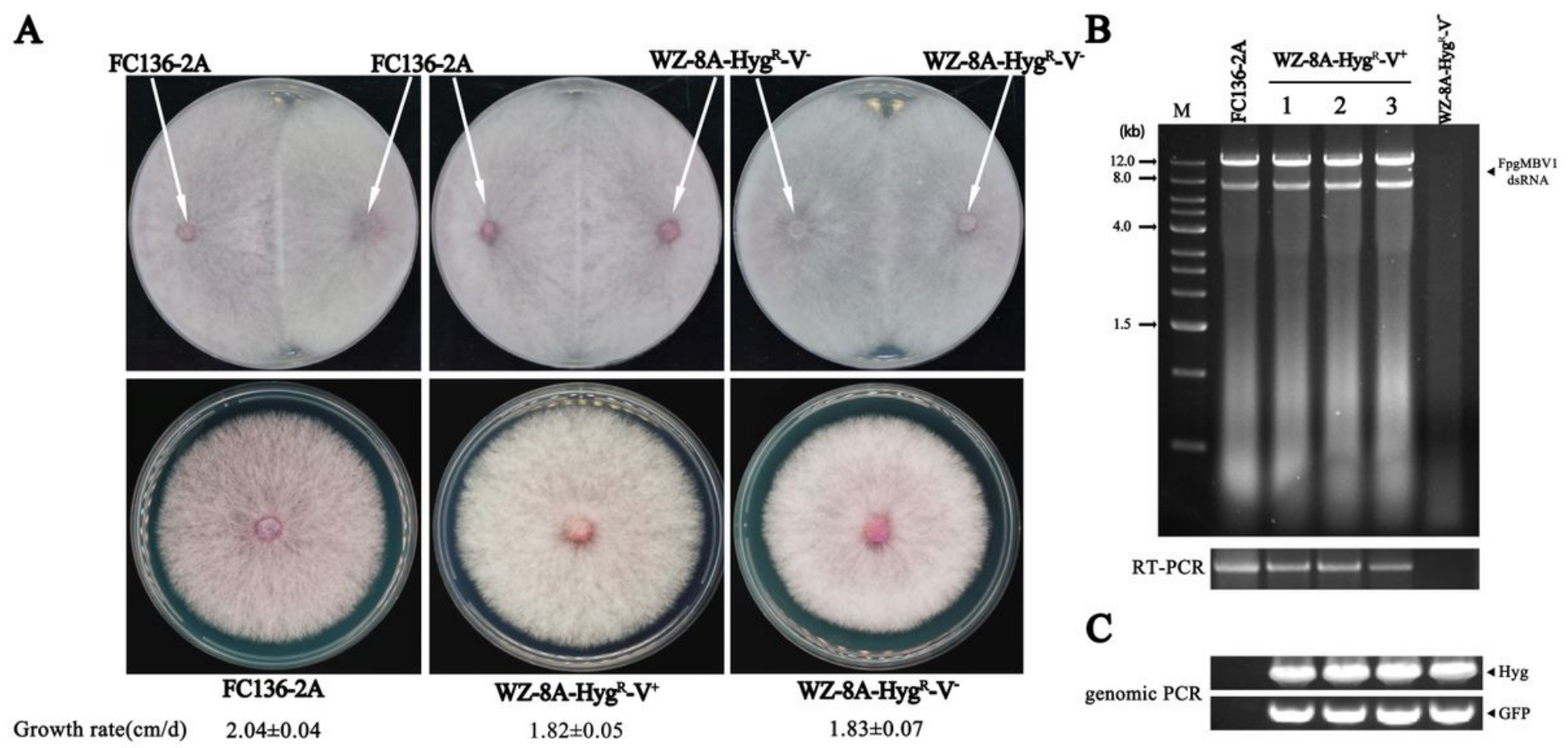

\section{Figure 3}

Horizontal transferring of FpgMBV1 from FC136-2A to WZ-8A-HygR-V-. (A): Pairing culture of FC136-2A/ FC136-2A, FC136-2A/ WZ-8A-HygR-V- and WZ-8A-HygR-V-/ WZ-8A-HygR-V- (upper line), colonial morphology of FC136-2A, WZ-8A-HygR-V- and WZ-8A-HygR-V+ (lower line). (B): Detection of FpgMBV1 positive strains derived from pairing culture by agarose gel electrophoresis of dsRNA fractions (upper) and RT-PCR amplification using FpgMBV1-P3 specific primers (lower). (C) PCR validation of strains derived from pairing culture using Hph (upper) and GFP specific primers (lower). Lane M:1kb DNA Ladder Marker (TSINGKE). 

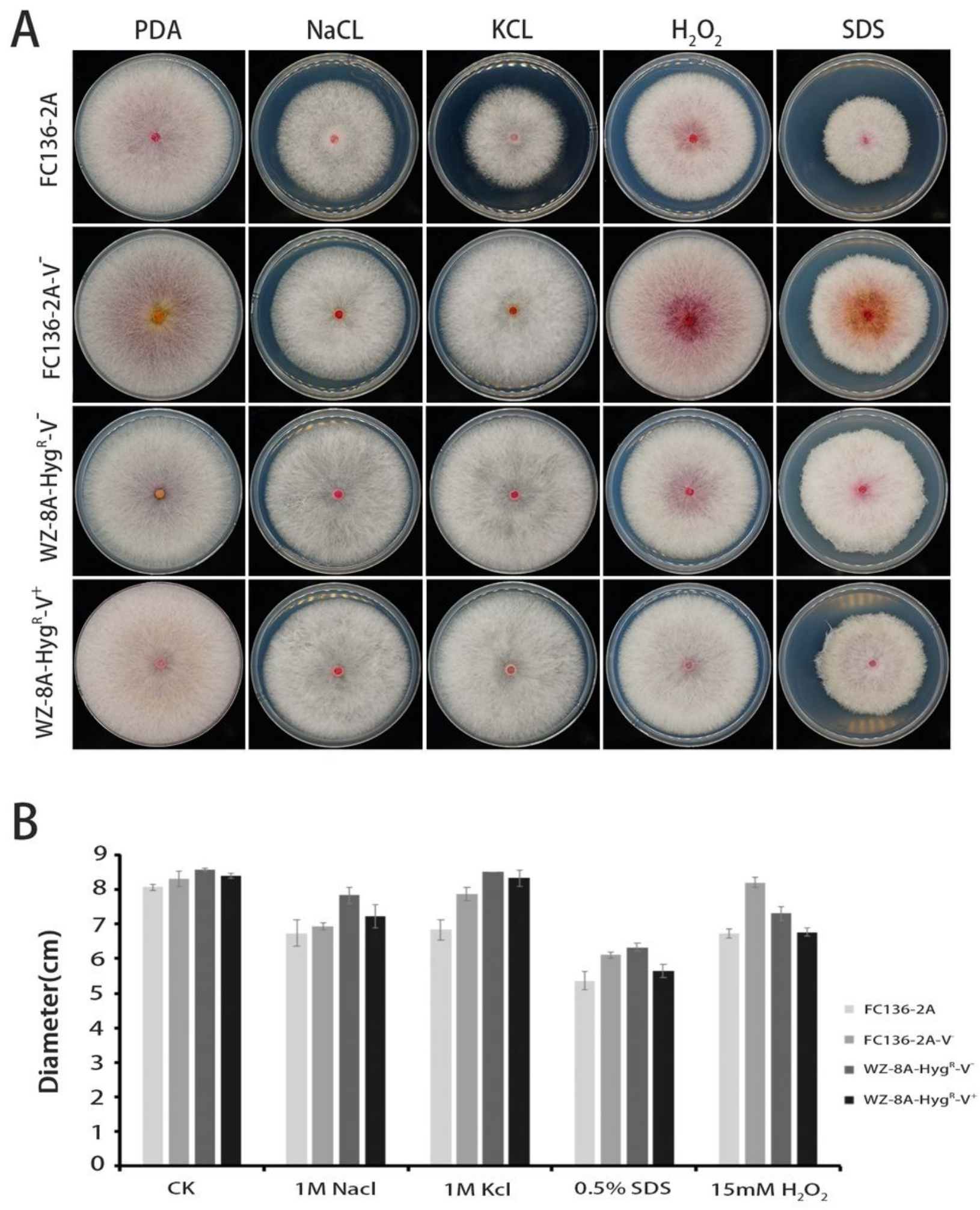

Figure 4

Colonial morphology (A) and growth rates (B) of F. pseudograminearum strains, FC136-2A, FC136-2A-V-, WZ-8A-HygR-V- and WZ-8A-HygR-V+, on PDA medium plates individually adding $1 \mathrm{M} \mathrm{NaCl}$ or $1 \mathrm{M} \mathrm{KCl}$ or $0.5 \%$ SDS or $15 \mathrm{mM} \mathrm{H} 2 \mathrm{O} 2$. 


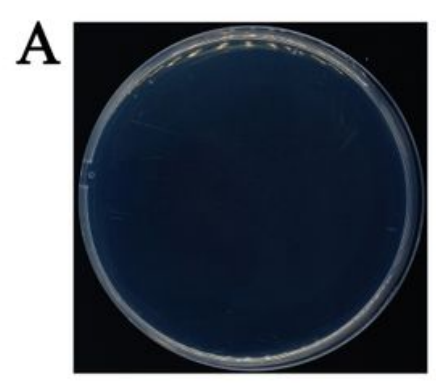

FC136-2A

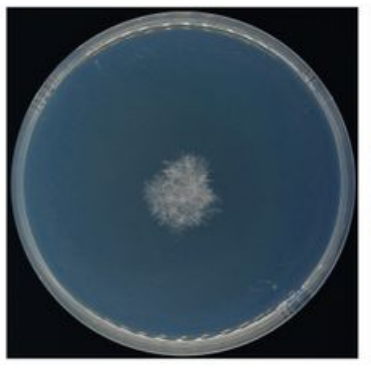

FC136-2A-V

B

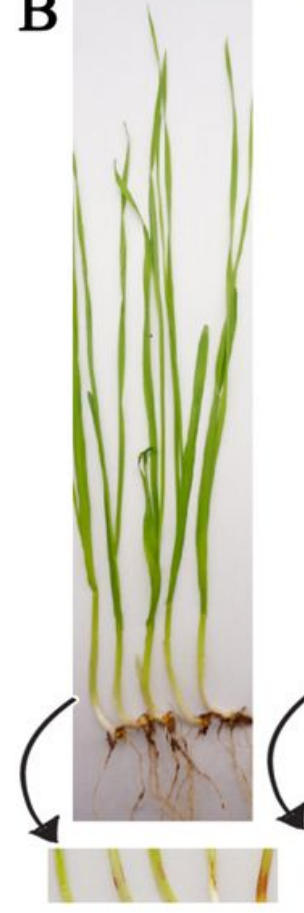

FC136-2A

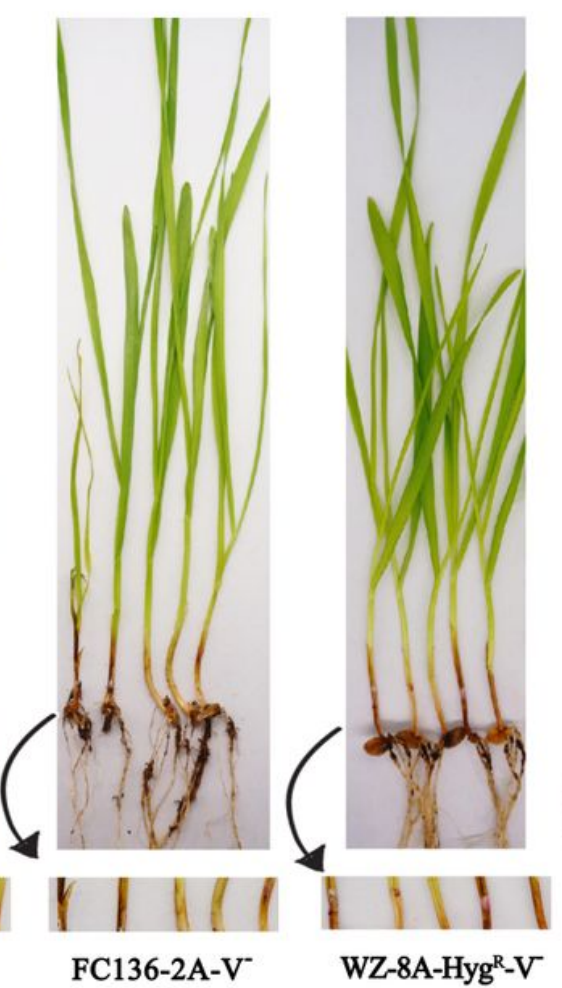

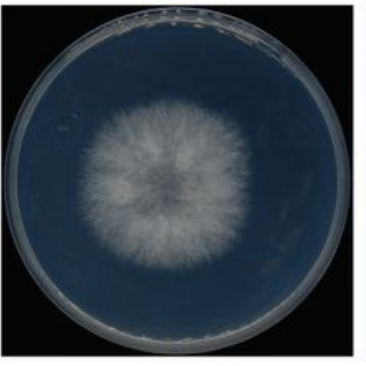

WZ-8A-Hyg ${ }^{\mathrm{R}}-\mathrm{V}$

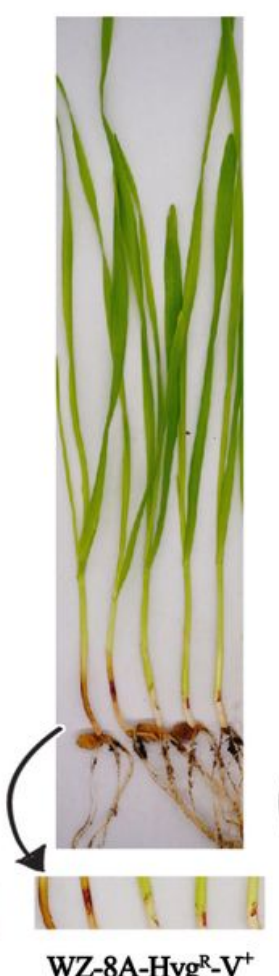

WZ-8A-Hyg ${ }^{\mathrm{R}}-\mathrm{V}^{+}$

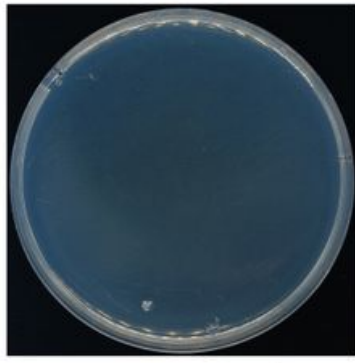

WZ-8A-Hyg ${ }^{\mathrm{R}}-\mathrm{V}^{+}$

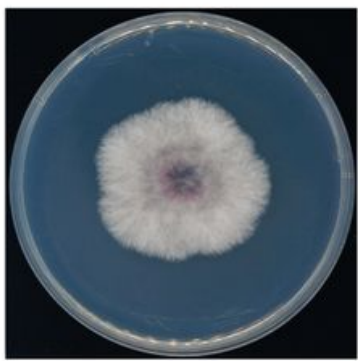

WZ-8A

C
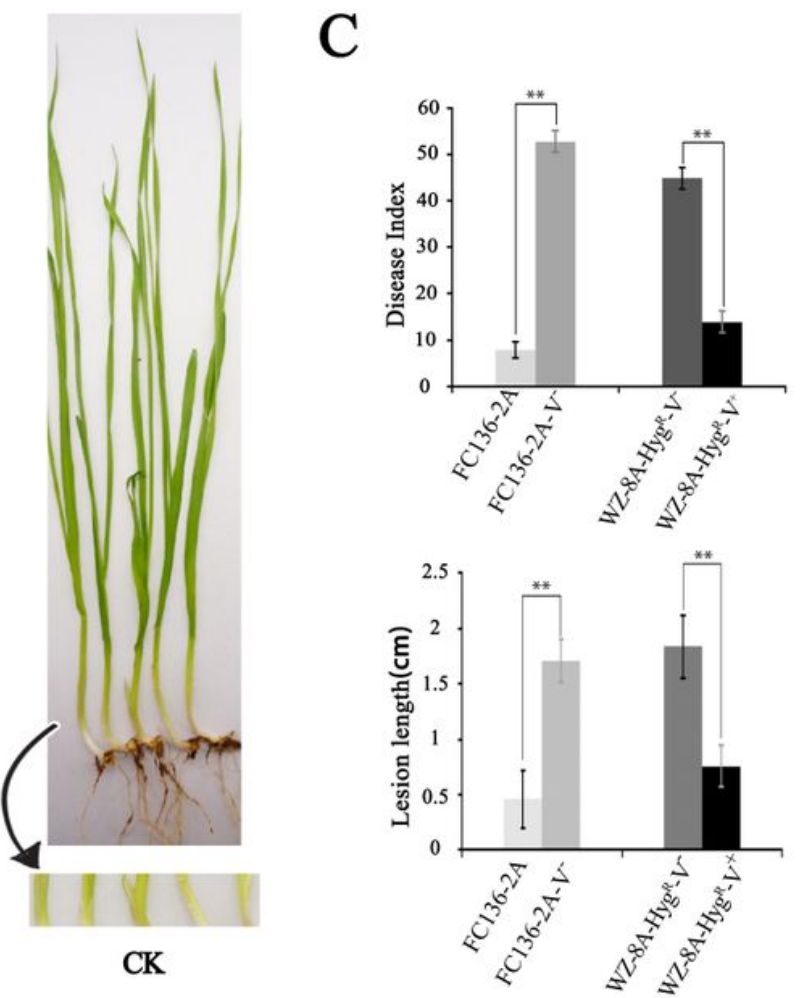

Figure 5

Colony formed in the cellophane pealed PDA plates (A) and pictures of wheat seedlings (upper in B) and the corresponding zoomed-in wheat crown parts (lower in B) and the disease index (upper in C) and lesion length (lower in $\mathrm{C}$ ) taken at 15 days after inoculation with $\mathrm{F}$. pseudograminearum strains WZ-8A, FC136-2A, FC136-2A-V-, WZ-8A-HygR-V- and WZ-8A-HygR-V+ on wheat seedlings of Aikang58 cultivar. 